This is an electronic reprint of the original article. This reprint may differ from the original in pagination and typographic detail.

Author(s): Liu, Wenbin; Neittaanmäki, Pekka; Tiba, Dan

Title: $\quad$ Existence for shape optimization problems in arbitrary dimension

Year: $\quad 2003$

Version:

Please cite the original version:

Liu, W., Neittaanmäki, P., \& Tiba, D. (2003). Existence for shape optimization problems in arbitrary dimension. SIAM Journal On Control and Optimization, 41(5), 1440-1454. https://doi.org/10.1137/s0363012901388142

All material supplied via JYX is protected by copyright and other intellectual property rights, and duplication or sale of all or part of any of the repository collections is not permitted, except that material may be duplicated by you for your research use or educational purposes in electronic or print form. You must obtain permission for any other use. Electronic or print copies may not be offered, whether for sale or otherwise to anyone who is not an authorised user. 


\title{
EXISTENCE FOR SHAPE OPTIMIZATION PROBLEMS IN ARBITRARY DIMENSION*
}

\author{
W. B. LIU $^{\dagger}$, P. NEITTAANMÄKI ${ }^{\ddagger}$, AND D. TIBA ${ }^{\S}$
}

\begin{abstract}
We discuss some existence results for optimal design problems governed by second order elliptic equations with the homogeneous Neumann boundary conditions or with the interior transmission conditions. We show that our continuity hypotheses for the unknown boundaries yield the compactness of the associated characteristic functions, which, in turn, guarantees convergence of any minimizing sequences for the first problem. In the second case, weaker assumptions of measurability type are shown to be sufficient for the existence of the optimal material distribution. We impose no restriction on the dimension of the underlying Euclidean space.
\end{abstract}

Key words. uniform segment property, compactness, existence of optimal shapers

AMS subject classifications. 49D37, 65K10

PII. S0363012901388142

1. Introduction. In this paper, we study existence for two shape optimization problems. The first is the following optimal shape design problem:

(SONB)

$$
\begin{array}{r}
\min _{\Omega \in \mathcal{O}} \int_{\Omega}\left(y-y_{d}\right)^{2} d x \\
-\Delta y+y=f,\left.\quad \frac{\partial y}{\partial n}\right|_{\partial \Omega}=0,
\end{array}
$$

where $\mathcal{O}$ is a class of admissible open sets inside a fixed open set $D$ in $\mathbf{R}^{m}$, and $f, y_{d} \in L^{2}(D)$.

The second problem is the following material distribution design problem:

$$
\begin{gathered}
\min _{\Omega \in \mathcal{O}} \int_{E \cap \Omega}\left|y_{1}-z_{d}\right|^{2} d x+\int_{E \cap(D \backslash \Omega)}\left|y_{2}-z_{d}\right|^{2} d x \\
-a_{1} \Delta y_{1}+b_{1} y_{1}=f \quad \text { in } \Omega, \\
-a_{2} \Delta y_{2}+b_{2} y_{2}=f \quad \text { in } D \backslash \bar{\Omega}, \\
a_{1} \frac{\partial y_{1}}{\partial n}=a_{2} \frac{\partial y_{2}}{\partial n}, \quad y_{1}=y_{2} \quad \text { in } \partial \Omega \backslash(\partial \Omega \cap \partial D), \\
a_{i} \frac{\partial y_{i}}{\partial n}=0 \quad \text { in } \Gamma_{1}, \quad y_{i}=0 \quad \text { in } \Gamma_{2}, \quad i=1,2,
\end{gathered}
$$

where $E \subset D$ are two given bounded domains in $\mathbf{R}^{m}, \Gamma_{1} \cup \Gamma_{2}=\partial D$ with $\Gamma_{1} \cap \Gamma_{2}=\emptyset$, $z_{d} \in L^{2}(E)$, and $\mathcal{O}$ is a class of admissible open sets inside $D$. The details of the above two problems will be specified in sections 3 and 4 .

It is well known that, in general, such shape optimization problems have no solutions without assuming further regularity conditions on the boundaries of the

* Received by the editors April 18, 2001; accepted for publication (in revised form) March 28, 2002; published electronically January 3, 2003.

http://www.siam.org/journals/sicon/41-5/38814.html

${ }^{\dagger}$ CBS, University of Kent, Canterbury, CT2 7PE, UK (W.B.Liu@ukc.ac.uk).

¥Department of Mathematical Information Technology, University of Jyväskylä, P.O. Box 35, FIN-40351 Jyväskylä, Finland (pn@mit.jyu.fi).

§Institute of Mathematics, Romanian Academy, P.O. Box 1-764, RO-70700 Bucharest, Romania (Dan.Tiba@imar.ro). 
domain classes; see Pironneau [20] for some counterexamples. Assuming the cone property on $\mathcal{O}$ uniformly, for example, one can prove that the above optimal shape design problem indeed has solutions; see Chenais [6] and Pironneau [20] for the details. Furthermore, much effort has been devoted in the scientific literature to the relaxation of the regularity conditions required for the boundaries of the unknown domains in optimal design problems. This question is discussed in detail in the monographs by Pironneau [20], Haslinger and Neittaanmäki [8], Sokolowski and Zolesio [21], and Tiba [24], for instance. As the range of optimal design problems is very wide, including as well control into coefficients problems, optimization of certain evolution systems, some problems originating in mechanics, etc., there is a rich variety of existence results of interest. We quote here just the recent papers by Sverak [23], Bucur and Zolesio $[4,3,5]$, and Henrot [9], where the question of the dependence of solutions of elliptic equations on the underlying domain of definition is discussed in a general setting and various sufficient compactness conditions are introduced. However, a complete solution of the problem seems not to be known, to our knowledge.

In this work, we first prove existence for the above optimal shape design problem governed by the Neumann boundary value problems, under the mere assumption that the unknown open sets are of class $\mathcal{C}$ (or, equivalently, they have the segment property - see Maz'ja [17] and Adams [1]) with some uniformity with respect to the parameters - see section 3 for the details. Our conditions allow cusps or certain oscillations of the boundaries, but cracks or oscillations dense in a set of positive measure (in the sense of Hausdorff-Pompeiu) are not permitted. Then, in section 4, it is shown that, for the material distribution problem, i.e., in the transmission boundary value problems, much weaker assumptions of measurability type are sufficient to obtain existence of the optimal sets. Moreover, all of our results are valid in any space dimension. This is an advantage over much of the existing literature, where very often the case of space dimension two is studied.

The approach that we are using is described in detail in section 2 and has its origin in our previous works-Liu [13], Liu and Rubio [15], Mäkinen, Neittaanmäki, and Tiba [16], and Neittaanmäki and Tiba [19]. Roughly speaking, we replace the extension technique for passing to the limit in the PDEs defined in a sequence of open sets by a local convergence analysis (see Lemma 3.2 and its proof). For set convergence, we introduce a concept of parametric convergence, which can be easily adapted to various possible representations of open sets and preserves some needed properties. As an example, the Hausdorff-Pompeiu convergence is a special case of the parametric convergence, choosing a certain distance function as the parametric representation. Notice that this is essentially different from the one used by Sverak [23]; see Proposition 2.5 and the subsequent remark.

It is recognized in the scientific literature that the a.e. convergence of the corresponding characteristic functions is an essential step in any convergence result for the PDEs defined in a sequence of open sets. Our treatment of this question, appearing mainly in sections 2 and 4 , is based on a new technique using the maximal monotone extension of the Heaviside mapping in $\mathbf{R} \times \mathbf{R}$ and the closure properties of monotone operators. We also propose, in this setting, a new approximation procedure for the characteristic functions by means of the Yosida approximation and of the Friedrichs mollifiers. In this respect, we point out the constructive character of our method. Some numerical experiments together with an approximation result are reported in Mäkinen, Neittaanmäki, and Tiba [16].

Finally, we mention that, in the recent paper by Sprekels and Tiba [22], some 
design problems, which are formulated as control into coefficients problems, are discussed. It is shown (by different methods) that the boundedness of the coefficients is sufficient to prove existence. An announcement of some of the results from the present work was published in Liu, Neittaanmäki, and Tiba [14] without proofs.

2. Convergence of open sets and of mappings. Let $A, B$ be two open sets contained in the bounded domain $D$ of $\mathbf{R}^{m}, m \in N$. The distance $\delta$ between $A$ and $B$ is defined by

$$
\begin{aligned}
& \rho(A, B)=\sup _{x \in \bar{D}-A} \inf _{y \in \bar{D}-B}\|x-y\|_{\mathbf{R}^{m}}, \\
& \delta(A, B)=\max \{\rho(A, B), \rho(B, A)\},
\end{aligned}
$$

and it is the Hausdorff-Pompeiu distance between the closed sets $\bar{D} \backslash A$ and $\bar{D} \backslash B$; see Pironneau [20] and Kuratowski [11]. We shall denote by Hlim the limit in the sense of Hausdorff-Pompeiu.

Another frequently used distance notion is

$$
\mu(A, B)=\operatorname{meas}[(A \backslash B) \cup(B \backslash A)],
$$

defined by the Lebesgue measure of the symmetric set difference between $A$ and $B$; see Hewitt and Stromberg [10, p. 144]. It should be noted that $\mu$ coincides with the well-known Ekeland metric in $L^{\infty}(D)$ applied to characteristic functions:

$$
d_{E}\left(\chi_{A}, \chi_{B}\right)=\operatorname{meas}\left\{x \in D \mid \chi_{A}(x) \neq \chi_{B}(x)\right\}=\mu(A, B) .
$$

Relations (2.3), (2.4) are defined up to sets of measure zero. Without supplementary regularity assumptions on the boundaries of the sets, there is no connection between $\delta$ and $\mu$. For instance, let $\overline{S(0,1)}$ be the closed unit ball in $\mathbf{R}^{m}$. Add $n$ (closed) rays of length 2 , starting from the origin, into the ball such that the union of the rays is dense in $\overline{S(0,2)}$ as $n \rightarrow \infty$, and denote the resulting (closed) sets by $A_{n}$. Then

$$
\begin{array}{ll}
\operatorname{Hlim}\left(A_{n}\right)=\overline{S(0,2)} & \text { for } n \rightarrow \infty, \\
\mu\left(A_{n}, \overline{S(0,1)}\right) \rightarrow 0 & \text { for } n \rightarrow \infty
\end{array}
$$

In Chenais [6], it was proved that, for uniformly Lipschitz domains, convergence in the metric (2.2) yields convergence in the metric (2.4) with the same limit (up to a set with zero measure).

Let us now introduce the mappings $d_{\Omega}: \bar{D} \rightarrow \mathbf{R}$, based on the Euclidean distance functions associated with the domain $\Omega$ and its complementary:

$$
d_{\Omega}(x)= \begin{cases}\operatorname{dist}(x, \bar{D} \backslash \Omega) & \text { if } x \in \Omega, \\ 0 & \text { if } x \in \partial \Omega, \\ -\operatorname{dist}(x, \bar{\Omega}) & \text { if } x \in \bar{D} \backslash \bar{\Omega} .\end{cases}
$$

The mapping $d_{\Omega}$ is uniformly Lipschitzian in $\bar{D}$ for any open subset $\Omega \subset \bar{D}$; see Clarke [7]. Let $\Omega_{n} \subset D$ be a sequence of open sets, not necessarily connected. Let $d_{n}=d_{\Omega_{n}}$ be the associated mappings via (2.7). By the Ascoli theorem, on a subsequence again denoted by $n$, we have $d_{n} \rightarrow \hat{d}$ uniformly in $\bar{D}$. However, $\hat{d}$ is not necessarily a function of the same type since, in general, the Hlim's of $\bar{\Omega}_{n}$ and of $\bar{D} \backslash \Omega_{n}$ may be not complementary to each other (see the above example with the sets $\left.A_{n}\right)$. Let $\widehat{\Omega}=\{x \in D \mid \hat{d}(x)>0\}$ (possibly void). 
Proposition 2.1.

$$
\widehat{\Omega}=D \backslash \operatorname{Hlim}\left(\bar{D} \backslash \Omega_{n}\right) .
$$

Proof. Let $x \in D \backslash \operatorname{Hlim}\left(\bar{D} \backslash \Omega_{n}\right)$ so that $x \notin \operatorname{Hlim}\left(\bar{D} \backslash \Omega_{n}\right)$. Then $\lim _{n \rightarrow \infty} \operatorname{dist}(x, \bar{D} \backslash$ $\left.\Omega_{n}\right)>0$. Thus $\lim _{n \rightarrow \infty} d_{n}(x)>0$; i.e., $x \in \widehat{\Omega}$.

Conversely, assume that $x \in \widehat{\Omega}$ and $x \notin D \backslash \operatorname{Hlim}\left(\bar{D} \backslash \Omega_{n}\right)$. Then $\hat{d}(x)>0$, and $x \in \operatorname{Hlim}\left(\bar{D} \backslash \Omega_{n}\right)$. That is, $\hat{d}(x)>0$, and there are $x_{n} \in \bar{D} \backslash \Omega_{n}$ such that $x_{n} \rightarrow x$. This means that $\hat{d}(x)>0, d_{n}\left(x_{n}\right) \leq 0$, and $x_{n} \rightarrow x$. By the uniform convergence, we have $\hat{d}(x)>0$ and $\hat{d}(x) \leq 0$, which lead to a contradiction. It follows that $\widehat{\Omega}=D \backslash \operatorname{Hlim}\left(\bar{D} \backslash \Omega_{n}\right)$, which is the desired conclusion.

Remark. The above proposition shows that the well-known compactness property of the Hausdorff-Pompeiu distance is a direct consequence of the Ascoli compactness criteria. A variant of the mapping $d_{\Omega}$ (identically zero outside $\Omega$ ) was considered by Sverak [23], who also proved a result similar to Proposition 2.1.

Proposition 2.2. If $\operatorname{Hlim}\left(\bar{D} \backslash \Omega_{n}\right)=\bar{D} \backslash \widehat{\Omega}$, then, for any compact $\mathcal{K} \subset \widehat{\Omega}$, there is an $n_{\mathcal{K}}=n(\mathcal{K}) \in N$ such that $\mathcal{K} \subset \Omega_{n}$ for $n \geq n_{\mathcal{K}}$.

Proof. We use the same notation as in Proposition 2.1. Since $\hat{d}$ is continuous on $\bar{D}$ and strictly positive on $\mathcal{K}$, there is a $c_{\mathcal{K}}>0$ such that

$$
\hat{d}(x) \geq c_{\mathcal{K}}>0 \quad \forall x \in \mathcal{K}
$$

By the uniform convergence, for $n \geq n_{\mathcal{K}}$, we obtain $d_{n}(x) \geq \frac{1}{2} c_{\mathcal{K}}>0$ for all $x \in \mathcal{K}$. That is, $\mathcal{K} \subset \Omega_{n}$ for $n \geq n_{\mathcal{K}}$, as required.

Remark. This property is called the $\Gamma$-property by Liu [13] and Liu and Rubio [15], and it plays an essential role in the local convergence theory for the solutions of PDEs defined in sequences of bounded domains. The same property is also proved in Pironneau [20], by different methods, together with other domain convergence results.

DEFINITION 2.3. We say that the sequence of open sets $\Omega_{n} \subset D$ is parametrically convergent to the open set $\widetilde{\Omega} \subset D$ if there is a sequence of continuous mappings $p_{n}: \bar{D} \rightarrow \mathbf{R}$ such that $p_{n} \rightarrow \tilde{p}$ uniformly in $\bar{D}$ and

$$
\begin{aligned}
\Omega_{n} & =\left\{x \in D \mid p_{n}(x)>0\right\}, \\
D \backslash \bar{\Omega}_{n} & =\left\{x \in D \mid p_{n}(x)<0\right\}, \\
\widetilde{\Omega} & =\{x \in D \mid \tilde{p}(x)>0\}, \\
D \backslash \overline{\widetilde{\Omega}} & =\{x \in D \mid \tilde{p}(x)<0\} .
\end{aligned}
$$

We denote the limit by $\widetilde{\Omega}=p-\lim \Omega_{n}$.

Remark. The "parametrization" $p_{n}$ associated with the domain $\Omega_{n}$ is not unique, and the distance mapping $d_{n}$ is just one example. The $p$-limit and the convergence properties depend on the parametrization. If it is different from the function $d_{\Omega}$, then the convergence may differ from the Hausdorff-Pompeiu convergence. For instance, we choose $\check{p}: \mathbf{R} \rightarrow \mathbf{R}$ by

$$
\check{p}(x)= \begin{cases}-(x-1)^{2}+\frac{1}{2}, & x \geq \frac{1}{2} \\ x^{2}, & |x| \leq \frac{1}{2} \\ -(x+1)^{2}+\frac{1}{2}, & x \leq-\frac{1}{2}\end{cases}
$$


and we rotate its graph to define a continuous mapping $p: \mathbf{R}^{2} \rightarrow \mathbf{R}$. Take $p_{n}: \mathbf{R}^{2} \rightarrow$ $\mathbf{R}, p_{n}(x)=p(x)+\frac{1}{n}$. Then the corresponding domains are $\Omega_{n}=\left\{\left.x \in \mathbf{R}^{2}|| x\right|_{\mathbf{R}^{2}}<\right.$ $\left.1+\sqrt{\frac{n+2}{2 n}}\right\}$ and $\Omega=\left\{\left.x \in \mathbf{R}^{2}|0<| x\right|_{\mathbf{R}^{2}}<1+\frac{1}{\sqrt{2}}\right\}$. Notice that $\Omega$ is nonsmooth, $\Omega=p-\lim \Omega_{n}$, and $\bar{\Omega} \neq \operatorname{Hlim} \bar{\Omega}_{n}$. If $\check{p}$ is zero around $x=0$ on some interval, then $p-\lim \Omega_{n}$ will be a circular crown, etc. By taking $\sup \left(p_{n}, p_{k}\right)$ or $\inf \left(p_{n}, p_{k}\right)$, one can easily "parametrize" $\Omega_{n} \cup \Omega_{k}$ or $\Omega_{n} \cap \Omega_{k}$.

Proposition 2.4. The parametric convergence has the $\Gamma$-property for any parametrization.

Proof. This is similar to the proof of Proposition 2.2.

Remark. It is possible to weaken the conditions in Definition 2.3 by replacing the uniform convergence with other types of functional convergence for the mapping $p_{n}$. This will be used in section 4 (see Theorem 4.1 and its subsequent remarks).

Proposition 2.5. If $\Omega=p-\lim \Omega_{n}$ and the closed set $C=\{x \in \bar{D} \mid \tilde{p}(x)=0\}$ has zero measure, then $\chi_{\Omega_{n}} \rightarrow \chi_{\Omega}$ a.e. in $D$.

Proof. If $x \in \Omega$, then $\tilde{p}(x)>0$ so that $p_{n}(x)>0$ for $n \geq n_{x}$ (depending on $x$ ). Thus $\chi_{\Omega_{n}}(x)=\chi_{\Omega}(x)=1$ for $n \geq n_{x}$. If $x \in D \backslash \bar{\Omega}$, then $\tilde{p}(x)<0$ and $p_{n}(x)<0$ for $n \geq n_{x}$; i.e., $x \in D \backslash \bar{\Omega}_{n}$ for $n \geq n_{x}$. Consequently, $\chi_{\Omega_{n}}(x)=\chi_{\Omega}(x)=0$ for $n \geq n_{x}$.

As the set $C$ has zero measure, we get that $\chi_{\Omega_{n}}(x) \rightarrow \chi_{\Omega}(x)$ a.e. in $D$.

Remark. The family of distance-type mappings used by Sverak [23] does not satisfy this property.

DEFinition 2.6. Assume that $\Omega=p-\lim \Omega_{n}$, and let $y_{n} \in H^{1}\left(\Omega_{n}\right)$ be such that $\left\{\left|y_{n}\right|_{H^{1}\left(\Omega_{n}\right)}\right\}$ is bounded. We say that $\left\{y_{n}\right\}$ is locally convergent to $y \in H^{1}(\Omega)$, and we write $y=L-\lim y_{n}$ if, for any $G \subset \subset \Omega$ (open set compactly embedded in $\Omega$ ), we have

$$
\left.\left.y_{n}\right|_{G} \longrightarrow y\right|_{G} \quad \text { weakly in } H^{1}(G)
$$

Remark. This definition is motivated by Proposition 2.4. The limit mapping $y$ is uniquely determined. The convergence in (2.8) is also valid in $L^{2}(G)$ strongly for any $G \subset \subset \Omega$.

TheOREM 2.7 (compactness). Assume $\Omega=p-\lim \Omega_{n}$. Suppose that $y_{n} \in$ $H^{1}\left(\Omega_{n}\right)$ and $\left|y_{n}\right|_{H^{1}\left(\Omega_{n}\right)}$ is uniformly bounded. Then there are $a y \in H^{1}(\Omega)$ and $a$ subsequence still denoted by $y_{n}$ such that $y=L-\lim y_{n}$.

Proof. Take a sequence $G_{j} \subset \subset \Omega$ such that $G_{j} \subset G_{j+1}$ and $\bigcup G_{j}=\Omega$. For each $j$, we take subsequences (one after another and all denoted by $n$ ) such that $\left.y_{n}\right|_{G_{j}} \longrightarrow y^{j}$ weakly in $H^{1}\left(G_{j}\right)$. We define $y$ on $\Omega$ by $y(x)=y^{j}(x)$ a.e. $x \in G_{j}$, which is possible by the properties of $\left\{G_{j}\right\}_{j \in \mathcal{N}}$. Clearly, $y \in L^{2}(\Omega)$ since $\left|y_{n}\right|_{L^{2}\left(G_{j}\right)}$ is uniformly bounded with respect to $n$ and $j$. Consider any $\varphi \in \mathcal{D}(\Omega)$. There is a $j_{0}$ such that $\varphi \in \mathcal{D}\left(G_{j}\right)$ for all $j \geq j_{0}$. Therefore,

$$
\int_{G_{j}} \nabla y \varphi=\int_{\Omega} \nabla y \varphi=-\int_{\Omega} y \nabla \varphi=-\int_{G_{j}} y^{j} \nabla \varphi=\int_{G_{j}} \nabla y^{j} \varphi .
$$

This yields that $\nabla y=\nabla y^{j}$ in $G_{j}$ for all $j \geq j_{0}$. As $\left|y^{j}\right|_{H^{1}\left(G_{j}\right)}$ is bounded with respect to $j$, we obtain that $\nabla y \in L^{2}(\Omega)^{m}$; i.e., $y \in H^{1}(\Omega)$. Relation (2.8) then follows, and the proof is completed.

TheOREM 2.8 (lower semicontinuity). If $l: \mathbf{R}^{m} \times \mathbf{R} \times \mathbf{R}^{m} \rightarrow \mathbf{R}$ is nonnegative and measurable, $l(x, \cdot, \cdot)$ is continuous on $\mathbf{R} \times \mathbf{R}^{m}, l(x, s, \cdot)$ is convex on $\mathbf{R}^{m}$, and 
$\Omega=p-\lim \Omega_{n}$, then

$$
\int_{\Omega} l(x, y, \nabla y) d x \leq \liminf _{n \rightarrow \infty} \int_{\Omega_{n}} l\left(x, y_{n}, \nabla y_{n}\right) d x
$$

provided that $y=L-\lim y_{n}$.

Proof. Let $\left\{G_{j}\right\}$ be selected as in the previous proof. Then we have $\chi_{G_{j}} \rightarrow \chi_{\Omega}$ a.e. in $D$. For any fixed $G_{j}$, we have that $y_{n} \rightarrow y$ weakly in $H^{1}\left(G_{j}\right)$, and we obtain

$$
\int_{G_{j}} l(x, y, \nabla y) d x \leq \liminf _{n \rightarrow \infty} \int_{G_{j}} l\left(x, y_{n}, \nabla y_{n}\right) d x
$$

since weak lower semicontinuity is a well-known property of the convex integrals in the fixed domains. Next, Fatou's lemma gives

$$
\begin{aligned}
\int_{\Omega} l(x, y, \nabla y) d x & =\int_{\Omega} \lim _{j \rightarrow \infty} \chi_{G_{j}} l(x, y, \nabla y) d x \\
& \leq \liminf _{j \rightarrow \infty} \int_{G_{j}} l(x, y, \nabla y) d x \\
& \leq \liminf _{j \rightarrow \infty} \liminf _{n \rightarrow \infty} \int_{\Omega_{n}} l\left(x, y_{n}, \nabla y_{n}\right) d x \\
& =\liminf _{n \rightarrow \infty} \int_{\Omega_{n}} l\left(x, y_{n}, \nabla y_{n}\right) d x .
\end{aligned}
$$

The positivity of $l$ is essential in the above proof.

Remark. Theorems 2.7 and 2.8 are variants or results previously proved by Liu and Rubio [15] and Liu [13]. It should be noted that it is enough to assume the $\Gamma$-property for the open sets $\Omega_{n}$ and $\Omega$ to prove Theorems 2.7 and 2.8 .

3. Equicontinuity. We consider the model problem (SONB). The problem is formulated as

$$
\min _{\Omega} \int_{\Omega}\left(y-y_{d}\right)^{2} d x
$$

subject to the following variational equation with the homogeneous Neumann boundary condition:

$$
\int_{\Omega} \nabla y \nabla v+\int_{\Omega} y v=\int_{\Omega} f v \quad \forall v \in H^{1}(\Omega)
$$

where $\Omega$ is a variable open set such that $\Omega \subset D$ with $D$ being a fixed bounded open set in $\mathbf{R}^{m}$, and $y_{d} \in L^{2}(D)$. For the admissible class of open sets denoted by $\mathcal{O}$, we require that they have the $\mathcal{C}$-property (or, equivalently, the segment property) with some uniform constants:

(H1) We consider a family $\mathcal{F}$ of equibounded and equiuniformly continuous functions $g: S(0, k) \rightarrow \mathbf{R}$, with $k>0$ fixed and $S(0, k) \subset \mathbf{R}^{m-1}$ an open ball. For any $\Omega \in \mathcal{O}$, there is a subset $\mathcal{F}_{\Omega} \subset \mathcal{F}$, and, for any $g \in \mathcal{F}_{\Omega}$, we associate an orthogonal system of axes of center $o_{g} \in \partial \Omega$, "vertical" vector $l_{g} \in \mathbf{R}^{m}$ of unit length, and a rotation $R_{g}$ in $\mathbf{R}^{m}$ such that $l_{g}=R_{g}(0,0, \ldots, 0,1)$ and

$$
\bigcup_{g \in \mathcal{F}_{\Omega}}\left\{R_{g}(s, 0)+o_{g}+g(s) l_{g} \mid s \in S(0, k)\right\}=\partial \Omega
$$


(H2) There is an $a>0$ such that, for any $\Omega \in \mathcal{O}$ and any $g \in \mathcal{F}_{\Omega}$, the uniform segment property is valid:

$$
\begin{array}{ll}
R_{g}(s, 0)+o_{g}+(g(s)+t) l_{g} \in \mathbf{R}^{m} \backslash \bar{\Omega} & \forall s \in S(0, k), \forall t \in] 0, a[, \\
R_{g}(s, 0)+o_{g}+(g(s)-t) l_{g} \in \Omega & \forall s \in S(0, k), \forall t \in] 0, a[.
\end{array}
$$

These two conditions represent the usual definition of boundaries of class $\mathcal{C}$ with added uniformity assumptions. Notice that, due to the compactness of $\partial \Omega$, it can be covered by a finite number of local charts; therefore, both conditions are automatically satisfied, and the only real requirement is the uniformity with respect to the whole family $\mathcal{O}$, which does not allow the local charts to shrink.

Our specific requirement is that there is a constant $r \in] 0, k[$ such that

$$
\bigcup_{g \in \mathcal{F}_{\Omega}}\left\{R_{g}(s, 0)+o_{g}+g(s) l_{g} \mid s \in \overline{S(0, r)}\right\}=\partial \Omega
$$

for any $\Omega \in \mathcal{O}$. This is a uniform restriction (or extension) property for the whole family $\mathcal{F}$ of the local charts. It avoids, for instance, clustering of singularities (like cusps) near the boundary of any local chart. Notice that, due to the finite numbers of the local charts, a positive number $0<r_{\Omega}<k$ can be found in each $\Omega$ with the above property. We just assume that it can be chosen independent of $\Omega$.

Example. Take $\left.g(x)=x^{\frac{1}{2}}, x \in\right]-\alpha, \alpha$, to be a local chart with Hölder regularity for some domain in $\mathbf{R}^{2}$. In $x=0$, we have a cusp, and the segment property is fulfilled only by the vertical segments. Thus the segment choice for local charts with cusps is unique, and only cusps with the same "axis" may belong to the same local chart. Hypothesis (H3) requires, in particular, that cusps with different "axes" do not cluster. In the common part (which cannot shrink) of neighboring local charts, no cusp can occur.

Remark. In the counterexample of Pironneau [20], with infinitely many oscillations of the boundary in a rectangular region, all of the conditions in (H1)-(H3) are fulfilled except the equicontinuity of the local charts. This shows the essential importance of this assumption, reflected by the title of the section. Examples of continuous oscillating boundaries which are not even of class $\mathcal{C}$ may be found in the book by Maz'ja [17]. It is also known that domains with cuts are not of class $\mathcal{C}$. Notice, however, that our assumptions allow infinitely many oscillations with vanishing amplitude (to preserve equicontinuity).

THEOREM 3.1 (compactness and existence). Let $\left\{\Omega_{n}\right\}$ be a minimizing sequence of open sets for the problem (3.1) satisfying the assumptions (H1)-(H3). Then there is an open set $\widehat{\Omega}$ of class $\mathcal{C}$ which is a solution of the problem (3.1) and which satisfies (H1)-(H3). Furthermore, $\chi_{\Omega_{n}} \rightarrow \chi_{\hat{\Omega}}$ a.e.

Proof. We may assume that $\bar{D}$ is large enough to include $\Omega_{n}$ and the segments defined in (H2). Denote by $d_{n}$ the distance-type functions introduced in (2.7) corresponding to $\Omega_{n}$ and by $\hat{d}$ their uniform limit, $\hat{d} \in C(\bar{D})$. Let $\Lambda=\{x \in$ $\bar{D} \mid \hat{d}(x) \geq 0\}$ be a closed set, which is clearly nonvoid. Take $\hat{x} \in \Lambda$ such that $\hat{d}(x)=0$. Then $d_{n}(\hat{x}) \rightarrow 0$, by the definition of $\hat{d}$. By the definition of $d_{n}$, there are $x_{n} \in \partial \Omega_{n}, x_{n} \rightarrow \hat{x}$ (and $d_{n}\left(x_{n}\right)=0$ ). By (H3), there are $g_{n} \in \mathcal{F}_{\Omega_{n}}$ such that $x_{n}=R_{g_{n}}\left(s_{n}, 0\right)+o_{g_{n}}+g_{n}\left(s_{n}\right) l_{g_{n}}, d_{n}\left(o_{g_{n}}\right)=0$, and $s_{n} \in \overline{S(0, r)}$. Under our conditions, we may assume that $s_{n} \rightarrow \hat{s} \in \overline{S(0, r)}$ and $g_{n} \rightarrow \hat{g}$ uniformly in $\overline{S(0, k)}$, with $\hat{g}$ being continuous in $\bar{D}, R_{g_{n}} \rightarrow \widehat{R}, o_{g_{n}} \rightarrow \hat{o}$ with $\hat{d}(\hat{o})=0, l_{g_{n}} \rightarrow \hat{l}$ as matrices or 
vectors (since all are bounded) with $\widehat{R}(0,0, \ldots, 0,1)=\hat{l}$, and $|\hat{l}|=1$. We have

$$
\begin{gathered}
\hat{x}=\lim x_{n}=\lim \left(R_{g_{n}}\left(s_{n}, 0\right)+o_{g_{n}}+g_{n}\left(s_{n}\right) l_{g_{n}}\right)=\widehat{R}(\hat{s}, 0)+\hat{o}+\hat{g}(\hat{s}) \hat{l}, \\
d_{n}\left(R_{g_{n}}(s, 0)+o_{g_{n}}+g_{n}(s) l_{g_{n}}\right) \rightarrow \hat{d}(\widehat{R}(s, 0)+\hat{o}+\hat{g}(s) \hat{l})=0 \quad \forall s \in S(0, k) .
\end{gathered}
$$

We show the segment property.

Take any $\varepsilon \in] 0, a\left[\right.$, and consider the point $\widehat{R}(s, 0)+\hat{o}+(\hat{g}(s)-\varepsilon) \hat{l} \in \mathbf{R}^{m}$. We have that $R_{g_{n}}(s, 0)+o_{g_{n}}+\left(g_{n}(s)-\varepsilon\right) l_{g_{n}} \rightarrow \widehat{R}(s, 0)+\hat{o}+(\hat{g}(s)-\varepsilon) \hat{l}$; that is, $\widehat{R}(s, 0)+\hat{o}+(\hat{g}(s)-\varepsilon) \hat{l} \in \bar{D}$ for $s \in S(0, k)$. As $R_{g_{n}}(s, 0)+o_{g_{n}}+\left(g_{n}(s)-\varepsilon\right) l_{g_{n}} \in \Omega_{n}$ by (H2), we have $d_{n}\left(R_{g_{n}}(s, 0)+o_{g_{n}}+\left(g_{n}(s)-\varepsilon\right) l_{g_{n}}\right)>0$ for $\left.s \in S(0, k), \varepsilon \in\right] 0, a[$, $n \geq 1$. It follows that $\hat{d}(\widehat{R}(s, 0)+\hat{o}+(\hat{g}(s)-\varepsilon) \hat{l}) \geq 0$ for $s \in S(0, k), \varepsilon \in] 0, a[$; i.e., $(\widehat{R}(s, 0)+\hat{o}+(\hat{g}(s)-\varepsilon) \hat{l}) \in \Lambda$ for such values of the parameters $s, \varepsilon$.

For the outside segment property, a sharper estimate is needed. By the equicontinuity of $g_{n}$, there is an $\delta>0$ (depending only on $\varepsilon$ and independent of $s \in S(0, k)$ or $n \in N$ ) such that

$$
\left|g_{n}(t)-g_{n}(s)\right|<\frac{\varepsilon}{2} \quad \forall n, \forall t \in S(s, \delta) \cap S(0, k) .
$$

Then, for $\varepsilon<\frac{2}{3} a$, we get

$$
\begin{aligned}
& \operatorname{dist} {\left[R_{g_{n}}(s, 0)+o_{g_{n}}+\left(g_{n}(s)+\varepsilon\right) l_{g_{n}}, \partial \Omega_{n}\right] } \\
& \geq \min \left\{\frac{\varepsilon}{2}, \delta, a-\frac{3}{2} \varepsilon, \operatorname{dist}(s, \partial S(0, k)\} .\right.
\end{aligned}
$$

Here we use the uniform outside segment property for $\Omega_{n}$, i.e., $R_{g_{n}}(s, 0)+o_{g_{n}}+$ $\left(g_{n}(s)+\varepsilon\right) l_{g_{n}} \in D \backslash \bar{\Omega}_{n}$, for all $s \in S(0, k)$ and for all $\left.\varepsilon \in\right] 0, a[$. The inequality (3.6) comes from (3.5), which simply says that the cylinder $[S(0, k) \cap S(s, \delta)] \times\left[g_{n}(s)+\right.$ $\left.\frac{\varepsilon}{2}, g_{n}(s)+a-\frac{\varepsilon}{2}\right]$ after translation $o_{g_{n}}$ and rotation $R_{g_{n}}$ cannot intersect $\partial \Omega_{n}$ for any $n$. And the right-hand side in (3.6) estimates from below the distance between $\left(s, g_{n}(s)+\varepsilon\right)$ and the boundary of this cylinder. (This point is inside the cylinder for $\varepsilon<\frac{2}{3} a$.)

Then it yields

$$
d_{n}\left(R_{g_{n}}(s, 0)+o_{g_{n}}+\left(g_{n}(s)+\varepsilon\right) l_{g_{n}}\right) \leq-\min \left\{\frac{\varepsilon}{2}, \delta, a-\frac{3}{2} \varepsilon, \operatorname{dist}(s, \partial S(0, k))\right\} .
$$

Inequality (3.7) is independent of $n$, and we can take the limit, by the uniform convergence, to obtain

$$
\hat{d}(\widehat{R}(s, 0)+\hat{o}+(\hat{g}(s)+\varepsilon) \hat{l}) \leq-\min \left\{\frac{\varepsilon}{2}, \delta, a-\frac{3}{2} \varepsilon, \operatorname{dist}(s, \partial S(0, k))\right\} ;
$$

that is, $\hat{d}(\widehat{R}(s, 0)+\hat{o}+(\hat{g}(s)+\varepsilon) \hat{l})<0$ for all $s \in S(0, k)$ and for all $\varepsilon \in] 0, \frac{2}{3} a[$, and, consequently, $(\widehat{R}(s, 0)+\hat{o}+(\hat{g}(s)+\varepsilon) \hat{l}) \notin \Lambda$ for these values of the parameters $s, \varepsilon$. By choosing a smaller $\delta>0$, if necessary, we can replace $\frac{\varepsilon}{2}$ by $\frac{\varepsilon}{l}, l \in N$, and $\frac{3}{2} \varepsilon$ by $\frac{l+1}{l} \varepsilon$ in inequalities (3.5)-(3.8). Finally, we have that $\widehat{R}(s, 0)+\hat{o}+(\hat{g}(s)+\varepsilon) \hat{l} \notin \Lambda$ for $s \in S(0, k)$ and $\varepsilon \in] 0, a[$.

Notice that estimates like (3.8) can also be obtained for $\hat{d}(\widehat{R}(s, 0)+\hat{o}+(\hat{g}(s)-\varepsilon) \hat{l})$, $s \in S(0, k), \varepsilon \in] 0, a[$, with the reversed sign. Then

$$
\widehat{\Omega}=\{x \in D \mid \hat{d}(x)>0\}=\operatorname{int} \Lambda
$$


is a nonvoid open subset of $D$. The above argument shows that $\partial \widehat{\Omega}=\{x \in D \mid \hat{d}(x)=$ $0\}$ is of class $\mathcal{C}$ and satisfies (H1)-(H3) with the same constants $a, r, k$ and the same modulus of continuity.

By Proposition 2.1, we see that $\widehat{\Omega}=D \backslash \operatorname{Hlim}\left(\bar{D} \backslash \Omega_{n}\right)$ (and $\widehat{\Omega}=p-\lim \Omega_{n}$ ), which proves the compactness of the family $\mathcal{O}$.

Let us also remark that, by Proposition 2.5, we have $\chi_{\Omega_{n}} \rightarrow \chi_{\hat{\Omega}}$ a.e. in $D$, and, by the Lebesgue theorem, this convergence is valid in any $L^{q}(D), q \geq 1$. Here we also use the fact that $\partial \widehat{\Omega}=\{x \in D \mid \hat{d}(x)=0\}$ has zero measure in $\mathbf{R}^{m}$ since it can be represented as a finite union of graphs of continuous functions.

The fact that $\widehat{\Omega}$ is a solution of the problem (3.1) follows from the subsequent lemma and Theorem 2.8 .

LEMMA 3.2. Let $y_{n}, \hat{y}$ denote the unique solutions of (3.2) associated with $\Omega_{n}, \widehat{\Omega}$. Then $\hat{y}=L-\lim y_{n}$ on a subsequence.

Proof. Clearly, $y_{n} \in H^{1}\left(\Omega_{n}\right), \hat{y} \in H^{1}(\widehat{\Omega})$, and $\left\{\left|y_{n}\right|_{H^{1}\left(\Omega_{n}\right)}\right\}$ is bounded. By Proposition 2.4, for any open set $G \subset \subset \widehat{\Omega}$, there are $n_{G}$ such that $G \subset \Omega_{n}, n \geq n_{G}$. We have

$$
\int_{G}\left(\nabla y_{n} \nabla v+y_{n} v\right)-\int_{D} \chi_{\Omega_{n}} f v=\int_{\Omega_{n} \backslash G}\left(\nabla y_{n} \nabla v+y_{n} v\right) \quad \forall v \in C^{1}(\bar{D}) .
$$

We can estimate

$$
\left|\int_{\Omega_{n} \backslash G}\left(\nabla y_{n} \nabla v+y_{n} v\right)\right| \leq|v|_{C^{1}(\bar{D})}\left|y_{n}\right|_{H^{1}\left(\Omega_{n}\right)} \mu\left(\Omega_{n}-G\right)^{\frac{1}{2}} .
$$

Taking the limit $n \rightarrow \infty$ in (3.11), we have that (3.10) yields

$$
\left|\int_{G} \nabla \tilde{y} \nabla v+\int_{G} \tilde{y} v-\int_{\widehat{\Omega}} f v\right| \leq M|v|_{C^{1}(\bar{D})} \mu(\widehat{\Omega}-G)^{\frac{1}{2}}
$$

(due to the convergence of the characteristic functions of $\Omega_{n}$ ), where $\tilde{y}$ denotes the $L$-limit of $y_{n}$ given by Theorem 2.7. We can take an increasing sequence of open sets $G_{j} \subset \subset \widehat{\Omega}$ such that $\cup G_{j}=\widehat{\Omega}$ and (3.12) gives

$$
\int_{\widehat{\Omega}} \nabla \tilde{y} \nabla v+\int_{\hat{\Omega}} \tilde{y} v=\int_{\widehat{\Omega}} f v \quad \forall v \in C^{1}(\bar{D}) .
$$

Since $\widehat{\Omega}$ has the segment property, $C^{1}(\bar{D})$ is dense in $H^{1}(\widehat{\Omega})$ (see Adams [1]) and (3.13) shows that $\tilde{y}=\hat{y}$. This ends the proof.

The semicontinuity result from Theorem 2.8 ensures that $\widehat{\Omega}$ is the desired minimizer for (3.1).

Remark. The above proof does not use the uniform extension property for functions in $H^{1}(\Omega)$. We replace it by a density property, which is, in fact, an approximate extension result. This is one of the reasons that we can renounce the cone property for $\partial \Omega$ and use the segment property instead. A more general cost functional, as in (2.9), may be considered in the problem (3.1).

Remark. If we impose uniform Hölder conditions for the family $\mathcal{O}$, the limit domain will satisfy a similar Hölder property. In this case, trace theorems are known (see, e.g., Ladyzenskaya and Uraltseva [12], Pironneau [20]), and the result of Theorem 3.1 can be then extended to Dirichlet boundary value problems. 
Example. A special simple case of the family $\mathcal{O}$ is obtained when global representations are given by

$$
\begin{aligned}
& \mathcal{O}_{1}=\left\{\Omega_{g} \mid g \in \mathcal{F}_{1}\right\}, \\
& \Omega_{g}=\{(s, \lambda) \in D \mid \lambda<g(s)\},
\end{aligned}
$$

where $D=U \times] 0, b\left[, U \subset \mathbf{R}^{m-1}\right.$ open domain, and $\mathcal{F}_{1}=\left\{g: U \rightarrow R_{+} \mid 0<c \leq g \leq b\right.$ in $U\}$.

By Theorem 3.1, one can immediately prove the following corollary.

Corollary 3.3. Assume that the family $\mathcal{F}_{1}$ is equicontinuous. Then the associated problem (3.1) has at least one solution in $\mathcal{O}_{1}$.

Proof. We indicate a direct argument. The family $\mathcal{F}_{1}$ is equibounded by definition. We take a minimizing sequence of domains $\Omega_{n}$ associated with $g_{n}$. We may assume that $g_{n} \rightarrow \hat{g}$ uniformly in $\bar{U}$ and, clearly, $\hat{g} \in \mathcal{F}_{1}$. Here it is possible to define the continuous mappings on $\bar{D}, p_{n}(s, \lambda)=g_{n}(s)-\lambda, \hat{p}(s, \lambda)=\hat{g}(s)-\lambda$, and $p_{n} \rightarrow \hat{p}$ uniformly; $\Omega_{n}=\left\{(s, \lambda) \in D \mid p_{n}(s, \lambda)>0\right), \widehat{\Omega}=\{(s, \lambda) \in D \mid \hat{p}(s, \lambda)>0\}$. Finally, $\widehat{\Omega}=p-\lim \Omega_{n}$, and the results of section 2 may be used directly to end the proof without using the distance functions.

Remark. Another family of domains of interest may be obtained by considering a domain $D \subset \mathbf{R}^{m}$ such that $D \supset B(0,1)$ (the unit ball) and then defining

$$
\mathcal{O}_{2}=\{\Omega \subset D \mid \Omega=h(B(0,1))\},
$$

where $h: D \rightarrow D$ is any homeomorphism, i.e., $h$ and $h^{-1}$ are continuous. This would be a generalization of the mapping method of Murat and Simon [18], where the mappings $h$ were assumed diffeomorphisms.

If $\Omega_{h}=h_{n}(B)$ and $h_{n} \rightarrow h, h_{n}^{-1} \rightarrow h^{-1}$ uniformly in $D$, and $\Omega=h(B)$, then it is easy to see that these domains have the $\Gamma$-property, and simple representation formulae are valid for $\Omega=h h_{n}^{-1}\left(\Omega_{n}\right), \partial \Omega=h h_{n}^{-1}\left(\partial \Omega_{n}\right)$. However, examples from Maz'ja [17] show that, conceptually, $\partial \Omega$ may not be of class $\mathcal{C}$ even when $\partial \Omega_{n}$ satisfies this assumption. Also, the compactness of $\mathcal{O}_{2}$ is not clear.

Remark. The examples of the families $\mathcal{O}_{1}$ and $\mathcal{O}_{2}$ also indicate that the concept of parametric convergence has enough flexibility to take into account various representation methods of open sets.

4. Measurability. We consider the material distribution problem (SOTB). In this case, it makes sense and is of interest to consider the case where the sets occupied by each material are merely measurable.

We fix $E \subset D$ to be two given bounded domains in $\mathbf{R}^{m}$ and $\Omega \subset D$ to be a variable measurable subset in some prescribed class $\mathcal{O}$, occupied by one material, while $D \backslash \Omega$ is occupied by another material. Then the physical properties of the two regions are different, and this is expressed by the fact that different coefficients appear in the elliptic equations describing the problem, which (formally) read as

$$
\begin{gathered}
-a_{1} \Delta y_{1}+b_{1} y_{1}=f \quad \text { in } \Omega, \\
-a_{2} \Delta y_{2}+b_{2} y_{2}=f \quad \text { in } D \backslash \bar{\Omega}, \\
a_{1} \frac{\partial y_{1}}{\partial n}=a_{2} \frac{\partial y_{2}}{\partial n}, \quad y_{1}=y_{2} \quad \text { in } \partial \Omega \backslash(\partial \Omega \cap \partial D), \\
a_{i} \frac{\partial y_{i}}{\partial n}=0 \quad \text { in } \Gamma_{1}, \quad y_{i}=0 \quad \text { in } \Gamma_{2}, \quad i=1,2,
\end{gathered}
$$


where $\Gamma_{1} \cup \Gamma_{2}=\partial D$ is assumed Lipschitz, $\Gamma_{1} \cap \Gamma_{2}=\emptyset, a_{i}, b_{i}>0, i=1,2$, are some constants, and $f \in L^{2}(D)$.

Let $y_{\Omega}$ be defined by

$$
y_{\Omega}(x)= \begin{cases}y_{1}(x) & \text { in } \Omega, \\ y_{2}(x) & \text { in } D \backslash \Omega .\end{cases}
$$

Then the weak formulation of the transmission problem (4.1)-(4.4) reads

$$
\begin{gathered}
\int_{D}\left\{\left[a_{1} \chi_{\Omega}+a_{2}\left(1-\chi_{\Omega}\right)\right] \nabla y_{\Omega} \cdot \nabla w+\left[b_{1} \chi_{\Omega}+b_{2}\left(1-\chi_{\Omega}\right)\right] y_{\Omega} w\right\} d x \\
=\int_{D} f w d x \quad \forall w \in V, \\
V=\left\{w \in H^{1}(D) \mid w=0 \quad \text { in } \Gamma_{2}\right\} .
\end{gathered}
$$

For any measurable subset $\Omega \subset D$, the bilinear form governing (4.6) is bounded and coercive in $V$, and there exists a unique solution $y_{\Omega} \in V$, which formally satisfies relations (4.1)-(4.5).

In Pironneau [20], by directly interpreting the characteristic function $\chi_{\Omega}$ as a control parameter, the following optimization problem is discussed:

$$
\min _{\Omega \in \mathcal{O}} \int_{E}\left|y_{\Omega}-z_{d}\right|^{2} d x
$$

subject to (4.6) and with some prescribed $z_{d} \in L^{2}(E)$. However, it is very difficult to impose the constraint that the control should take only the values 0 and 1 in the whole $D$.

Our approach is to specify the set $\mathcal{O}$ of admissible $\Omega$ by requiring that $\chi_{\Omega}$ is of the form $H\left(p_{\Omega}\right)$, where $p_{\Omega} \in U_{a d}$ (some admissible set of mappings will be defined later), and $H \subset \mathbf{R} \times \mathbf{R}$ is the maximal monotone extension of the Heaviside function:

$$
H(p)= \begin{cases}1, & p>0 \\ {[0,1],} & p=0 \\ 0, & p<0\end{cases}
$$

Notice that by taking, for instance, $p_{\Omega}=\chi_{\Omega}$, we have representations via $H$ for any measurable $\Omega$. If meas $(\partial \Omega)=0$, then we may take $p_{\Omega}=d_{\Omega}$, which is even Lipschitzian in $D$.

For the optimization problem (4.8), we define the class of admissible sets $\Omega$ by $\chi_{\Omega}=H\left(p_{\Omega}\right)$, where $p_{\Omega} \in U_{a d} \subset H_{\text {loc }}^{1}(D)$, and $p \in U_{a d}$ iff

$$
\begin{array}{rlrl}
|p|_{H^{1+\theta} \mathcal{K}(\mathcal{K})} & \leq M_{\mathcal{K}} & & \forall \mathcal{K} \subset \subset D, \theta_{\mathcal{K}}>0, \\
|p(x)|+|\nabla p(x)|_{\mathbf{R}^{m}} \geq \nu>0 & & \text { a.e. in } D .
\end{array}
$$

If meas $(\partial \Omega)=0$, the mappings $d_{\Omega}$ satisfy (4.11) (see Clarke [7, p. 66]) but do not satisfy (4.10) in general.

Conversely, the condition (4.11) ensures that the set

$$
\{x \in D \mid p(x)=0\}
$$

has zero measure for $p \in H_{\mathrm{loc}}^{1}(D)$; see Brezis [2, p. 195]. 
Remark. Under some stronger smoothness assumptions, condition (4.11) ensures the application of the implicit function theorem and a characterization of the "boundary" given by $\{x \in D \mid p(x)=0\}$. However, our regularity hypotheses are so weak that even the implicit theorem in Clarke [7, p. 255] for Lipschitzian mappings cannot be applied. The only properties that we have are (4.12) and the measurability of $\Omega$ 's, which are, in fact, defined up to sets of zero measure. Notice, as well, that the local character of (4.10) allows oscillations of $\partial \Omega$, even under the smoothness assumptions.

We reformulate the problem (4.6)-(4.8) as follows:

$$
\begin{gathered}
\min _{p \in U_{a d}} \int_{E}\left|y_{p}-z_{d}\right|^{2} d x \\
\int_{D}\left\{\left[a_{1} H(p)+a_{2}(1-H(p))\right] \nabla y_{p} \nabla w+\left[b_{1} H(p)\right.\right. \\
\left.\left.+b_{2}(1-H(p))\right] y_{p} w\right\} d x=\int_{D} f w d x \quad \forall w \in V .
\end{gathered}
$$

THEOREM 4.1. Under the hypotheses (4.10)-(4.11), the problem (4.13)-(4.14) has at least one optimal pair $[\bar{y}, \bar{p}] \in V \times U_{a d}$.

Proof. Obviously, $U_{a d} \neq \emptyset$ (as constant functions are in $U_{a d}$ ), and we may consider a minimizing sequence $\left[y_{n}, p_{n}\right] \subset V \times U_{a d}$. By taking an increasing sequence of open sets $\mathcal{K}_{l} \subset D$ such that $\cup \mathcal{K}_{l}=D$, and by the compactness of $\left\{p_{n} \mid \mathcal{K}_{l}\right\}$ in $H^{1}\left(\mathcal{K}_{l}\right)$, we may define $p \in U_{a d}$ such that $p_{n} \rightarrow p$ strongly in $H^{1}\left(\mathcal{K}_{l}\right)$ for all $l \in N, p_{n} \rightarrow p$, and $\nabla p_{n} \rightarrow \nabla p$ a.e. in $D$.

Since $a_{1} H\left(p_{n}\right)+a_{2}\left(1-H\left(p_{n}\right)\right) \geq c t>0$ and $b_{1} H\left(p_{n}\right)+b_{2}\left(1-H\left(p_{n}\right)\right) \geq c t>0$, it is easy to infer that $\left\{y_{n}\right\}$ is bounded in $V \subset H^{1}(D)$, and we may assume that $y_{n} \rightarrow y$ weakly in $H^{1}(D)$ on a subsequence.

Obviously, $H\left(p_{n}\right)$ is bounded in $L^{\infty}(D)$; therefore, we may also assume that $H\left(p_{n}\right) \rightarrow H(p)$ weakly star in $L^{\infty}(D)$. The identification of the limit is a consequence of the demiclosedness of the maximal monotone operator $H$, applied in $L^{2}(\mathcal{K}) \times L^{2}(\mathcal{K})$ for any $\mathcal{K} \subset \subset D$. Notice that, by $p \in U_{a d}$ and by (4.12), it follows that $H(p)$ is a characteristic function in $D$.

We also have that $H\left(p_{n}\right) \rightarrow H(p)$ a.e. in $D$. We know that $p_{n}(x) \rightarrow p(x) \neq 0$ a.e. in $D$. If $p(x)>0$, then $p_{n}(x)>0$ for $n \geq n_{x}$ and $H\left(p_{n}(x)\right)=H(p(x))=1$ for $n \geq n_{x}$. If $p(x)<0$, similarly, we obtain $H\left(p_{n}(x)\right)=H(p(x))=0$ for $n \geq n_{x}$. Consequently, we get that $H\left(p_{n}\right) \rightarrow H(p)$ strongly in $L^{s}(D)$ for all $s \geq 1$ by the Lebesgue dominated convergence theorem.

As $\left[y_{n}, p_{n}\right]$ satisfies (4.14), the above convergence allows to take the limit and to see that $[y, p]$ also satisfies (4.14) and $p \in U_{a d}$; i.e., the pair $[y, p]$ is admissible. Moreover, for the minimizing sequence, we have

$$
\lim _{n \rightarrow \infty} \int_{E}\left|y_{n}-z_{d}\right|^{2} d x=\int_{E}\left|y-z_{d}\right|^{2} d x
$$

which shows that the pair $[y, p]$ is optimal for the problem (4.13), (4.14), and we redenote it by $[\bar{y}, \bar{p}]$.

Remark. The above argument remains valid for any weakly lower semicontinuous cost functional on $H^{1}(D)$-for instance, for boundary cost functionals. Other boundary conditions may be imposed on $\partial D$ as well.

It is possible to impose (4.10) only for $\mathcal{K} \subset \subset D \backslash C$, where $C \subset D$ is a given closed set of zero measure. This allows cracks in the corresponding $\partial \Omega$ 's; see Bucur and Zolesio [3]. 
Remark. Considering the measurable sets $\Omega_{n}=\left\{x \in D \mid p_{n}(x)>0\right\}$, the proof of Theorem 4.1 uses a property of parametric convergence for $\Omega_{n}$ based on a.e. convergence in $D$ of $p_{n}$ and $\nabla p_{n}$, and a variant of Definition 2.3.

We continue by describing an approximation procedure which is suggested by our approach to characteristic functions. We denote by $H_{\varepsilon}$ the Yosida approximation of $H$, given in this case by

$$
H_{\varepsilon}(p)= \begin{cases}1, & p>\varepsilon, \\ \underline{p}, & p \in[0, \varepsilon], \\ 0, & p<0 .\end{cases}
$$

Notice that $H_{\varepsilon}$ is Lipschitzian with Lipschitz constant $\frac{1}{\varepsilon}$. The approximation of the optimization problem (4.13), (4.14) is obtained by replacing (4.14) with

$$
\begin{aligned}
\int_{D} & \left\{\left[a_{1} H_{\varepsilon}(p)+a_{2}\left(1-H_{\varepsilon}(p)\right)\right] \nabla y_{p}^{\varepsilon} \nabla w+\left[b_{1} H_{\varepsilon}(p)+b_{2}\left(1-H_{\varepsilon}(p)\right)\right] y_{p}^{\varepsilon} w\right\} d x \\
& =\int_{D} f w d x \quad \forall w \in V .
\end{aligned}
$$

By a variant of Theorem 4.1, we have existence of at least one optimal pair $\left[y_{\varepsilon}, p_{\varepsilon}\right] \in$ $H^{1}(D) \times U_{a d}$ for the problem (4.13), (4.14').

THEOREM 4.2. For any open set $\mathcal{K} \subset \subset D$, we have $\left[y_{\varepsilon}, p_{\varepsilon}\right] \rightarrow[\hat{y}, \hat{p}]$ in the weakstrong topology of $H^{1}(D) \times H^{1}(\mathcal{K})$ on a subsequence, and $[\hat{y}, \hat{p}]$ is an optimal pair for the problem (4.13), (4.14).

Proof. By (4.10), we have $p_{\varepsilon} \rightarrow \hat{p}$ strongly in $H^{1}(\mathcal{K})$ on a subsequence, and $p_{\varepsilon} \rightarrow \hat{p}, \nabla p_{\varepsilon} \rightarrow \nabla \hat{p}$ a.e. in $D$ for some $\hat{p} \in U_{a d}$. As $1 \geq H_{\varepsilon}(p) \geq 0$ a.e. in $D$, we also obtain that $\left\{y_{\varepsilon}\right\}$ is bounded in $H^{1}(D)$ by fixing $w=y_{\varepsilon}$ in (4.14'). We may assume that $y_{\varepsilon} \rightarrow \hat{y}$ weakly in $H^{1}(D)$ on a subsequence. Moreover, it is known from the theory of maximal monotone operators that $H_{\varepsilon}\left(p_{\varepsilon}\right) \rightarrow H(\hat{p})$ weakly in $L^{2}(\mathcal{K})$ for any $\mathcal{K} \subset \subset D$ since $\left\{H_{\varepsilon}\left(p_{\varepsilon}\right)\right\}$ is bounded in $L^{\infty}(D)$ and $p_{\varepsilon} \rightarrow \hat{p}$ strongly in $L^{2}(\mathcal{K})$, for instance. By the fact that $\hat{p} \in U_{a d}$ and by (4.12), (4.9), we know that $H(\hat{p})$ is a characteristic function in $D$. We can also prove the pointwise convergence of $H_{\varepsilon}\left(p_{\varepsilon}\right)$ a.e. in $D$. If $\hat{p}(x)>0$, then $p_{\varepsilon}(x)>\frac{1}{2} \hat{p}(x)$ for $\varepsilon<\varepsilon_{x}$; that is, $p_{\varepsilon}(x)>\varepsilon$ for $\varepsilon<\varepsilon_{x}$ and $H_{\varepsilon}\left(p_{\varepsilon}(x)\right)=H(\hat{p}(x))=1$ by (4.15). If $\hat{p}(x)<0$, then $p_{\varepsilon}(x)<0$ for $\varepsilon<\varepsilon_{x}$ and $H_{\varepsilon}\left(p_{\varepsilon}(x)\right)=H(\hat{p}(x))=0$ for $\varepsilon<\varepsilon_{x}$. These two situations are valid a.e. in $D$ by $\hat{p} \in U_{a d}$ and (4.12). Combining these with the Lebesgue theorem, we obtain that $H_{\varepsilon}\left(p_{\varepsilon}\right) \rightarrow H(\hat{p})$ strongly in $L^{s}(D)$ for all $s \geq 1$. Then we can take the limit in $\left(4.14^{\prime}\right)$ and infer that the pair $[\hat{y}, \hat{p}]$ is admissible for the problem (4.13), (4.14). To show that it is optimal, we note that

$$
\int_{E}\left|y_{\varepsilon}-z_{d}\right|^{2} d x \leq \int_{E}\left|y_{p}^{\varepsilon}-z_{d}\right|^{2} d x
$$

where $y_{p}^{\varepsilon}$ denotes the solution of (4.14') corresponding to some $p \in U_{a d}$. By an argument of the same type as above, we can prove that $y_{p}^{\varepsilon} \rightarrow y_{p}$ weakly in $H^{1}(D)$ on a subsequence, where $y_{p}$ is the solution of (4.14) associated with $p$. Taking the limit in (4.16) yields the optimality of $[\hat{y}, \hat{p}]$ in the problem (4.13)-(4.14) and completes the proof.

Corollary 4.3. On a subsequence, we have

$$
y_{\varepsilon} \rightarrow \hat{y} \quad \text { strongly in } H^{1}(D)
$$


where $\hat{y}$ is some optimal state for the problem (4.13)-(4.14).

Proof. There is a constant $c>0$ such that

$$
\begin{aligned}
c\left|y_{\varepsilon}-\hat{y}\right|_{H^{1}(D)}^{2} \leq & \int_{D}\left[a_{1} H_{\varepsilon}\left(p_{\varepsilon}\right)+a_{2}\left(1-H_{\varepsilon}\left(p_{\varepsilon}\right)\right)\right]\left|\nabla\left(y_{\varepsilon}-\hat{y}\right)\right|_{\mathbf{R}^{m}}^{2} d x \\
& +\int_{D}\left[b_{1} H_{\varepsilon}\left(p_{\varepsilon}\right)+b_{2}\left(1-H_{\varepsilon}\left(p_{\varepsilon}\right)\right)\right]\left(y_{\varepsilon}-\hat{y}\right)^{2} d x \\
= & \int_{D}\left\{\left[a_{1} H_{\varepsilon}\left(p_{\varepsilon}\right)+a_{2}\left(1-H_{\varepsilon}\left(p_{\varepsilon}\right)\right)\right] \nabla y_{\varepsilon} \nabla\left(y_{\varepsilon}-\hat{y}\right)\right. \\
& \left.\quad+\left[b_{1} H_{\varepsilon}\left(p_{\varepsilon}\right)+b_{2}\left(1-H_{\varepsilon}\left(p_{\varepsilon}\right)\right)\right] y_{\varepsilon}\left(y_{\varepsilon}-\hat{y}\right)\right\} d x \\
& -\int_{D}\left\{\left[a_{1} H_{\varepsilon}\left(p_{\varepsilon}\right)+a_{2}\left(1-H_{\varepsilon}\left(p_{\varepsilon}\right)\right)\right] \nabla \hat{y} \nabla\left(y_{\varepsilon}-\hat{y}\right)\right) \\
& \left.\quad+\left[b_{1} H_{\varepsilon}\left(p_{\varepsilon}\right)+b_{2}\left(1-H_{\varepsilon}\left(p_{\varepsilon}\right)\right)\right] \hat{y}\left(y_{\varepsilon}-\hat{y}\right)\right\} d x \\
= & \int_{D} f\left(y_{\varepsilon}-\hat{y}\right) d x-\int_{D}\left\{\left[a_{1} H_{\varepsilon}\left(p_{\varepsilon}\right)\right.\right. \\
\left.\left.\quad+a_{2}\left(1-H_{\varepsilon}\left(p_{\varepsilon}\right)\right)\right] \nabla \hat{y} \nabla\left(y_{\varepsilon}-\hat{y}\right)\right) & \left.+\left[b_{1} H_{\varepsilon}\left(p_{\varepsilon}\right)+b_{2}\left(1-H_{\varepsilon}\left(p_{\varepsilon}\right)\right)\right] \hat{y}\left(y_{\varepsilon}-\hat{y}\right)\right\} d x \\
= & I_{1}+I_{2} .
\end{aligned}
$$

By Theorem 4.2, we may assume that $I_{1} \rightarrow 0$ on a sequence as $\varepsilon \rightarrow 0$. For $I_{2}$, we first estimate the term

$$
\int_{D}\left[a_{1} H_{\varepsilon}\left(p_{\varepsilon}\right)+a_{2}\left(1-H_{\varepsilon}\left(p_{\varepsilon}\right)\right)\right] \nabla \hat{y} \nabla\left(y_{\varepsilon}-\hat{y}\right) d x
$$

which is the most difficult. We know that $\nabla \hat{y} \nabla\left(y_{\varepsilon}-\hat{y}\right)$ is weakly convergent in $L^{1}(D)$ and the coefficients $a_{1} H_{\varepsilon}\left(p_{\varepsilon}\right)+a_{2}\left(1-H_{\varepsilon}\left(p_{\varepsilon}\right)\right)$ are bounded in $L^{\infty}(D)$ and strongly convergent in $L^{s}(D)$ for all $s \geq 1$. On a subsequence, we may assume that $\left[a_{1} H_{\varepsilon}\left(p_{\varepsilon}\right)+a_{2}\left(1-H_{\varepsilon}\left(p_{\varepsilon}\right)\right)\right] \nabla\left(y_{\varepsilon}-\hat{y}\right) \rightarrow u$ weakly in $L^{2}(D)^{m}$. Egorov's theorem gives that $a_{1} H_{\varepsilon}\left(p_{\varepsilon}\right)+a_{2}\left(1-H_{\varepsilon}\left(p_{\varepsilon}\right)\right) \rightarrow a_{1} H(\hat{p})+a_{2}(1-H(\hat{p}))$ uniformly in $D_{\delta}$ for any $\delta>0$ and some measurable subset $D_{\delta} \subset D$ with meas $\left(D-D_{\delta}\right)<\delta$. Combining this with $\nabla\left(y_{\varepsilon}-\hat{y}\right) \rightarrow 0$ weakly in $L^{2}(D)$, we have that $u=0$ a.e. in $D$. Then the limit of the integral (4.19) is zero, and the same is true, by a similar reasoning, for $I_{2}$. Finally, (4.18) has limit zero, and this achieves the proof.

Remark. It is possible to further smooth $H_{\varepsilon}$ by means of a Friedrichs mollifier and to compute the gradient of the smoothed cost functional (4.13) with respect to $p \in U_{a d}$. This shows the constructive character of our approach presented in this paper. Numerical tests for the problem in this section were reported in Mäkinen, Neittaanmäki, and Tiba [16] together with an approximation result.

Remark. In Pironneau [20, p. 134], it is mentioned that, by taking $a_{2} \rightarrow 0, b_{2} \rightarrow 0$ in (4.14), the Neumann boundary value is approximated. Therefore, the results of this section may open a way to relax the continuity assumptions from section 3. A similar idea is possible for Dirichlet boundary value problems, which could be obtained by taking $a_{2} \rightarrow \infty, b_{2} \rightarrow \infty$. The hypotheses under which such passages to the limit can be performed are not clear yet. 


\section{REFERENCES}

[1] R. Adams, Sobolev Spaces, Academic Press, New York, 1975.

[2] H. BrezIS, Analyse fonctionelle. Théorie et applications, Masson, Paris, 1983.

[3] D. Bucur AND J. P. Zolesio, Continuité par rapport an domaine dans le probleme de Neumann, C. R. Acad. Sci. Paris Sér. I Math., 319 (1994), pp. 57-60.

[4] D. Bucur and J. P. Zolesio, Optimization de forme sous contrainte capacitaire, C. R. Acad. Sci. Paris Sér. I Math., 318 (1994), pp. 795-800.

[5] D. BuCUR AND J. P. Zolesio, Free boundary problems and density perimeter, J. Differential Equations, 126 (1996), pp. 224-243.

[6] D. ChEnAIS, On the existence of a solution in a domain identification problem, J. Math. Anal. Appl., 52 (1975), pp. 189-219.

[7] F. H. Clarke, Optimization and Nonsmooth Analysis, John Wiley and Sons, New York, 1983.

[8] J. Haslinger AND P. NeitTAanmäKI, Finite Element Approximation of Optimal Shape, $M a-$ terial and Topology Design, John Wiley and Sons, Chichester, UK, 1996.

[9] A. Henrot, Continuity with respect to the domain for the Laplacian: A survey, Control Cybernet., 23 (1994), pp. 427-443.

[10] E. Hewitt and K. Stromberg, Real and Abstract Analysis, Springer-Verlag, Berlin, 1965.

[11] K. Kuratowski, Introduction to Set Theory and Topology, Pergamon Press, Oxford, UK, 1961.

[12] O. Ladyzenskaya and N. Uraltseva, Linear and Quasilinear Elliptic Equations, Academic Press, New York, 1968.

[13] W. B. LiU, Optimal Shape Design for Variational Inequalities, Ph.D. thesis, University of Leeds, Leeds, UK, 1991.

[14] W. B. LiU, P. NeitTaAnmÄKI, AND D. TiBa, Sur les problèmes d'optimisation structurelle, C. R. Acad. Sci. Paris Sér. I Math., 331 (2000), pp. 101-106.

[15] W. B. LiU AND J. E. RuBIO, Local convergences and optimal shape design, SIAM J. Control Optim., 30 (1992), pp. 49-62.

[16] R. MÄKINEN, P. NEITTAANMÄKI, AND D. TIBA, On a fixed domain approach for a shape optimization problem, in Computational and Applied Mathematics II, W. F. Ames and P. J. van der Houwen, eds., North-Holland, Amsterdam, 1992, pp. 317-326.

[17] V. Maz'JA, Sobolev Spaces, Springer-Verlag, Berlin, 1985.

[18] F. Murat And J. Simon, Studies on Optimal Shape Design Problems, Lecture Notes in Control and Inform. Sci. 41, Springer-Verlag, Berlin, 1976.

[19] P. Neittaanmäkiand D. Tiba, Existence and Approximation in Optimal Shape Design Problems, Tech. report 6, Department of Mathematics, Laboratory of Scientific Computing, University of Jyväskylä, Jyväskylä, Finland, 1998.

[20] O. Pironneau, Optimal Shape Design for Elliptic Systems, Springer-Verlag, Berlin, 1984.

[21] J. Sokolowski AND J. P. Zolesio, Introduction to Shape Optimization, Springer-Verlag, Berlin, 1991.

[22] J. Sprekels AND D. TiBA, A duality approach in the optimization of beams and plates, SIAM J. Control Optim., 37 (1998), pp. 486-501.

[23] V. SveraK, On optimal shape design, J. Math. Pures Appl. (9), 72 (1993), pp. 537-551.

[24] D. TiBA, Lectures on the Control of Elliptic Systems, Lecture Notes 32, Department of Mathematics, University of Jyväskylä, Jyväskylä, Finland, 1995. 